\section{Bone Grafts and Dental Implants Clinical Practice and New Paradigms}

Authors: Alfred Seban, Patrick Bonnaud

Publisher: Biotech Dental Group

Language: English

ISSN: 979-10-699-0887-1

Edition: 1/e

Publish Year: 2017

Pages: 431, illustrated

Price: $135.00 €$

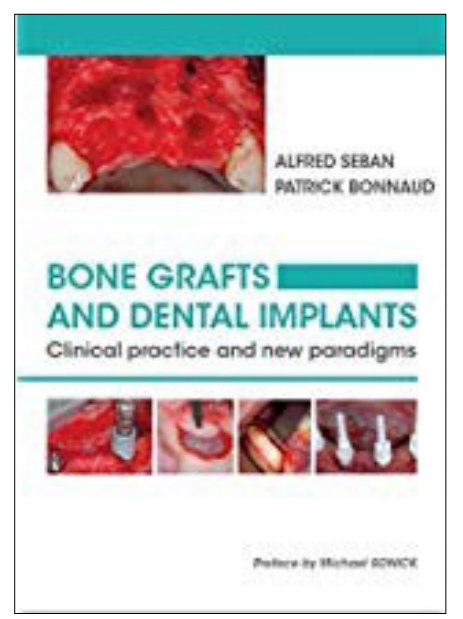

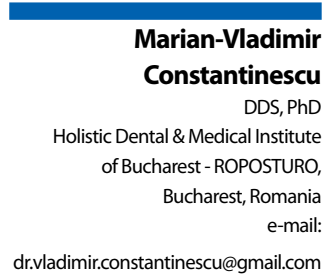

Nowdays, implants and bone grafts are used more frequently in each dental clinic dental, because the jaws and alveolar processes suffer resorption after tooth loss. The book entitled „Bone Grafts and Dental Implants: Clinical Practice and New Paradigms" by Alfred Seban and Patrick Bonnaud presents the experience and research accumulated over the years regarding tbone regeneration.

This textbook has six chapters.

Chapter 1 talks about the influence of systemic diseases, technologies and contemporary practices for bone grafts and implants. The effectiveness of a specific treatment for dental implants in extraction sockets, bone augmentation following dental avulsion and bone quality assessment are presented in chapter 2 .

The following chapter speaks about bone augmentation prior to the dental implant, the advantages and disadvanteges of graft materials, autologous bone grafts, corticocancelous allogenic bloc graft and operation technique (segmental osteotomy). Chapter 4 presents the maxillary sinus lift, the lateral and crestal approach, postoperative management and complication of the sinus lift. Chapter 5 approaches the clinical practice guidelines for bone grafting procedures prior to dental implant placement and describes 25 clinical cases.

The last chapter presents the preservation of biological width in implantology, while discussing one by one the concept of bone preservation, conical connection and biological width, narrow implants, short implants, full arch rehabilitation, immediate loading and biological width and immediate implant placement.

The authors succeed to present the most recent useful data in clinical practice as, graft materials, Bone Morphogenic Proteins, computer-guided implant placement, Cone Bean Computed Tomography for a complete approach of the practitioner. The book is an authentic guide to inform oral surgeons in bone graft and dental implants through various clinical cases illustrated with exceptional photos and radiographies associated with particular treatment protocols.

DOI: https://doi.org 10.25241/stomaeduj.2018.5(4).bookreview.1 